\title{
Anatomy is the key
}

\author{
Jon Karlsson
}

Published online: 10 December 2009

(c) Springer-Verlag 2009

In spite of several thousand of scientific publications concerned with the Anterior Cruciate Ligament (ACL), we are still struggling. And, without doubt, the "ACL mystery" is still not solved. The question is whether it ever will be.

Using "ACL" as a search word on Google, you will end up with more then 100,000 hits, some scientific, but most not. The overwhelming information is not based on science, like cadaver studies, or randomised controlled clinical trials. Why is this? And why can we, the scientists not solve the "ACL mystery" with all new studies? In this issue of the journal some of the best scientists in this field of research contribute and they have shed some new light on some of the research questions that are now in focus. The term "anatomic ACL reconstruction" is evolving and we understand more and more the importance of understanding the native anatomy of the ACL. This is crucial; anatomy and understanding of it is the key. A second term that has evolved alongside anatomic ACL reconstruction is "individualized surgery". When I started to practice orthopaedics a little more than 30 years ago, my first teacher always said ... "restore the anatomy"... This is truer now than ever.

Concerning "anatomic ACL reconstruction", it must be born in mind that there is no simple solution and that understanding the mechanism of ACL injury is not an easy task. Single-bundle or double-bundle is debated today, and none of these methods might be the optimal and only solution. Our belief is that ACL reconstruction-especially if performed early after the ACL injury-will lead to better kinematics, less morbidity, restoration of laxity and theoretically less risk of osteoarthritis in the long run. But, currently we have no solid proof of which technique is the best. This is where "anatomic ACL reconstruction" comes into play. The focus is to restore and reproduce the native anatomy of the ligament. This can be achieved by restoring the 2 bundles of the ACL, i.e. the functional units; anteromedial (AM) and postero-lateral (PL) bundles. This must be done by restoring the native insertion sites (foot-prints) by correct tunnel placement at the femur and tibia. This is often termed "insertion-site surgery". Tensioning of the different functional units must be ensured with the AMbundle taut throughout the entire range of motion, reaching maximum at approximately $45^{\circ}$ of knee flexion, while the PL-bundle is taut in near full extension. But, all these are technical issues that are of greatest importance. However, although good technique is necessary, understanding of the injury mechanism and the different anatomical requirements for each individual knee are even more important. That is why the principle of "individualized surgery" is what is needed. Each knee and each patient need to evaluated and understood in detail. The differences between knees must be emphasised. The most important issue is not single- or double-bundle; the road to success when it comes to ACL reconstruction is understanding of individuals' needs and the anatomy of their injured knees.

Once again; anatomy is the key.

J. Karlsson ( $₫)$

Department of Orthopaedics, Sahlgrenska University Hospital, Sahlgrenska Academy at Gothenburg University, Gothenburg, Sweden

e-mail: jon.karlsson@telia.com 